\title{
Thermographic measurement and thermal modelling of air gap inductors in H-F power forward converters
}

\author{
by R. Kasikowski*** , B. Więcek*, and M. Farrer**
}

\begin{abstract}
* Łódź Univ. of Technology, 90-924, Wólczańska Str., Łódź, Poland, rafal.kasikowski@stadiumpower.co.uk
\end{abstract}
** Stadium Power Ltd, Norwich Research Park, Norwich, United Kingdom, mike.farrer@stadiumpower.co.uk

\begin{abstract}
This paper examines the impact of the fringing field of an air gap on temperature distribution in the windings of output inductors in high-frequency forward converters. For this aim, a DC-DC H-F forward converter and a centre-gapped ferrite core inductor were constructed and the measurements of thermal characteristics were performed using dynamic, time-dependent thermographic analysis. The objective of this research was to consider how the length of an air gap affects the temperature distribution and power loss in the windings of output inductors in $\mathrm{H}-\mathrm{F}$ forward converters.
\end{abstract}

\section{Introduction}

Gapped ferrite core magnetic circuits are commonly used in a variety of power conversion applications. The introduction of an air gap into the magnetic path of the component brings about excess power loss in copper windings due to fringing magnetic field at the air gap. The lines of leaked magnetic flux cross the conductors inducing eddy currents that counteract the useful current, thus reducing the effective area of the conductor and increasing power loss. The impact of the fringe effect on temperature distribution in windings is well presented in figure 1 , where primary and secondary coils of a flyback transformer were wound on a gapped toroidal core. One can easily infer from the figure that the hottest spot is located directly above the air gap.

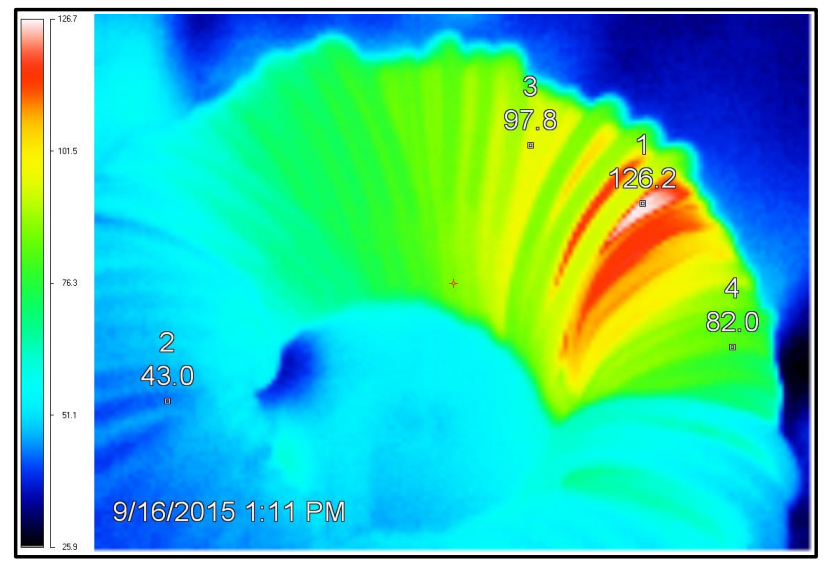

Fig. 1. Thermal image of a toroidal flyback transformer with an air gap

The ascertainment of high-frequency losses in windings affected by the fringing field is a complex problem and no simple method exists to estimate and extract the extra power dissipated due to fringing effect from a group of heat loss sources present in magnetic circuits. The fringing-effect losses are themselves affected by the number of factors such as the shape of the air gap, the distance from the gap to the windings, the size and geometry of the windings, the frequency of operation and so forth. This paper, in a simplified way, attempts to ascertain the increase of power loss brought out by the widening of the air gap.

Designing magnetic circuits without taking fringing field and its effects into consideration may lead to a multitude of problems. One of the key issues is localized heating caused by eddy currents generated within the windings observed in a form of so-called hot spots (see figure 1). This phenomena can be readily analysed by the utilization of IR thermography. Here the thermographic measurements and thermal impedance analysis of heat transfer in areas of the gapped output inductor windings affected by the fringing flux are presented. The distinctive pattern in temperature dispersion is observed and it is shown that the pattern is intrinsic to magnetics components with an air gap. 


\subsection{1/qirt.2016.053}

\section{Measurement setup}

To demonstrate the influence of the air gap size on the fringing-effect losses in wires inside the winding area as well as its impact on temperature distribution, the ferrite inductor of figure 2 has been used. The air gap is positioned symmetrically in the middle of the centre column and its length has been modified. The output inductor was constructed using ER28/14/11 3C90 ferrite cores [1] and mounted prior to the measurements on the back of $36 \mathrm{~W}$ forward converter (see figure 2) so as to eliminate the influence of any heat radiating top mounted components. The central columns of the cores were gapped to $1 \mathrm{~mm}$ and $2.5 \mathrm{~mm}$ and combine with a 24-turn coil wound on a phenolic bobbin to build two inductors of $87.08 \mu \mathrm{H}$ and $38.95 \mu \mathrm{H}$, respectively. The forward converter itself was set to run at the frequency of about $63.4 \mathrm{kHz}$.

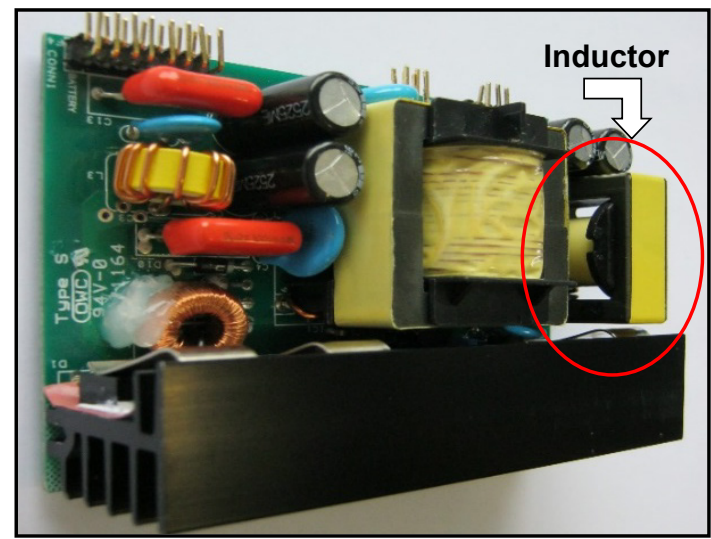

Fig. 2. Constructed forward converter (output inductor on far right)

The power loss in the inductor was ascertained by placing the very component in a sealed box and monitoring the increase in ambient temperature inside the box due to power dissipation. Subsequently, the choke was replaced with $100 \Omega$ resistor connected to a power source to reach the same increase in the ambient temperature, thus the power dissipated. The whole process was carried out in an air-conditioned laboratory at a constant ambient of $22^{\circ} \mathrm{C}$. at $25 \mathrm{~Hz}$ frame rate.

Thermographic measurements were carried out using a MWIR Cedip Titanium cooled photon camera operating

\section{Temperature distribution in centre-gapped core inductors}

Centre-gapped core inductors display a distinctive pattern in temperature distribution in windings if measured along the middle limb (see figure 3a). The ends of the coil show the lowest temperature while the maximum temperature is observed in the vicinity of the air gap. Although temperature distribution is almost certainly governed by a number of factors this pattern seems to be true for magnetic components with an air gap in the centre column with windings wound around it.

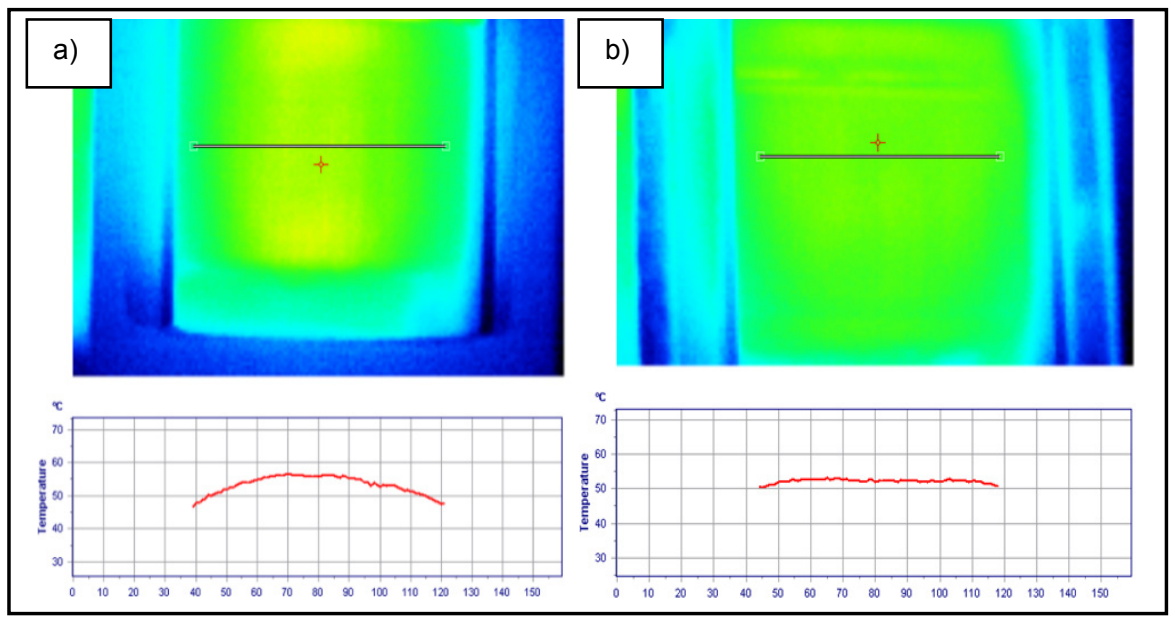

Fig. 3. Temperature distribution along the middle limb in a) gapped ferrite core b) ungapped iron powder core choke 
In order to demonstrate the above claim a single winding inductor was built on E42 bobbin. The coil was subsequently mounted on a $4 \mathrm{~mm}$ gap E42×21x15 ferrite core and an ungapped E168-26 iron powder core of the same size. Iron powder with a distributed air gap throughout the entire core has a high saturation point in comparison to ferrites and does not require a discrete air gap to prevent saturation in the conversion of relatively high power. The constructed chokes were placed in the forward converter which is a subject of this paper and run at the same frequency and at the same power level as the original. As shown in figure $3 \mathrm{~b}$ the temperature distribution in the iron powder core inductor measured along the middle limb does not exhibit the described above pattern so the pattern can fairly clearly be contributed to fringing-effect at the air gap.

\section{Thermal characteristics}

The thermographic analysis of the forward converter with ER28/14/113C90 ferrite core output chokes was started at the ambient temperature registered at the moment of switching on the converter and the entire measurement lasted 30 minutes for both $87.08 \mu \mathrm{H}$ and $38.95 \mu \mathrm{H}$ inductors. An exemplary thermal image extracted from the sequence recorded, a visual image and temperature distribution along the central leg are presented in figure 4.

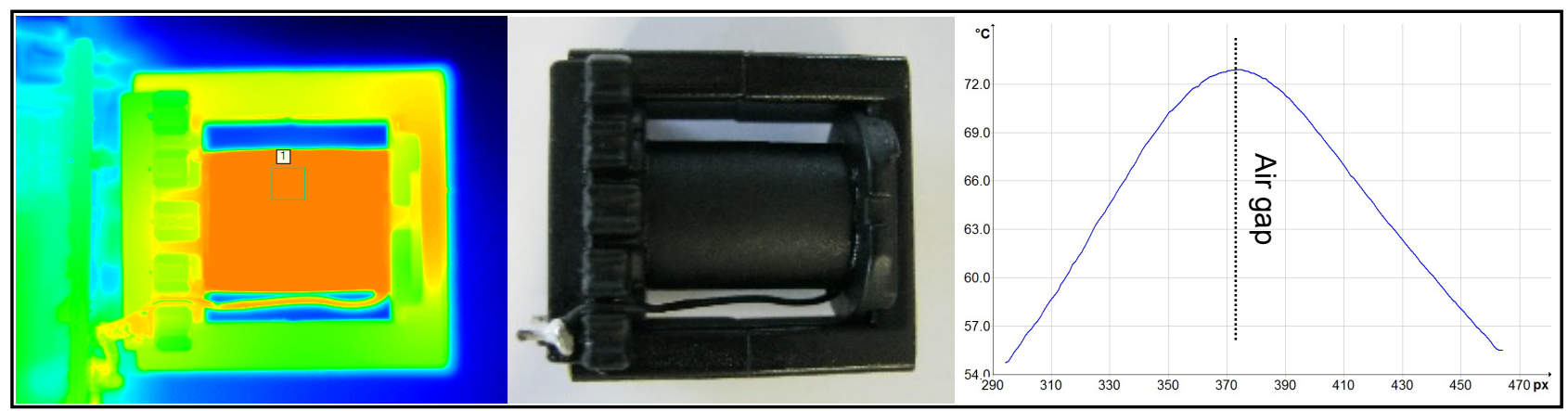

Fig. 4. ER28 core output inductor (thermal and visual image) and temperature distribution along central column

The results of the measurements at the area located over the air gap (see figure 4 - area 1) were converted to format compatible with T3STER software for thermal analysis [2]. The plots showing mean temperature rise over the ambient for each inductor configuration are shown below (figure 5).

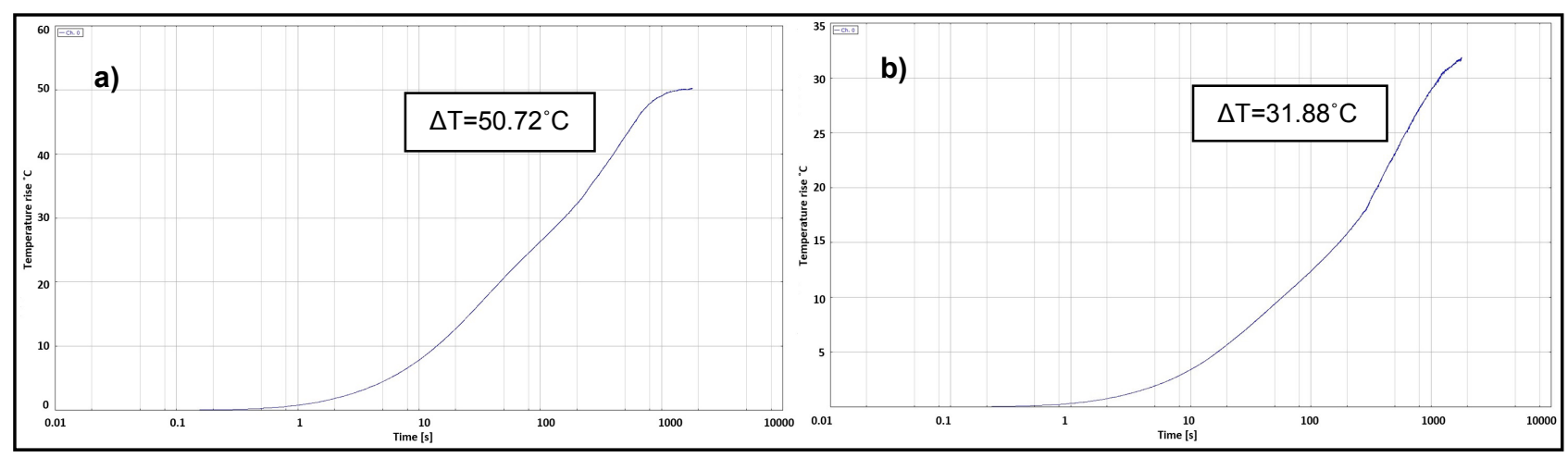

Fig. 5. Temperature rise over the ambient for a) $2.5 \mathrm{~mm}$ gap core and b) $1.0 \mathrm{~mm}$ gap core

As expected, higher temperature rise was recorded for the coil wounded on the core with $2.5 \mathrm{~mm}$ gap. One may argue that this was brought about by a number of factors with the fringing-effect as not necessarily a key source of additional dissipation. It is demonstrated in the sections below that causes such as potential changes in core loss and copper loss due to a higher current ripple cannot account entirely for the added loss. In consequence, one can conclude that it was an extra power loss due to the fringing flux effect.

The calculated complex thermal impedance can be represented in a form of Nyquist plots [3]. As presented in figure 6 the plots show two fairly clearly formed semi-circles with the third one protruding between the two. The number of semi-circles has a strong correlation with a time constant spectrum showing how many constants are there. The points where imaginary part of the thermal impedance reaches its extremal values correspond to the central frequencies of the semi-circles which are given by Eq. (1), where $T_{n}$ is an $\mathrm{n}^{\text {th }}$ time constant.

$$
\omega_{n}=\frac{1}{T_{n}}
$$




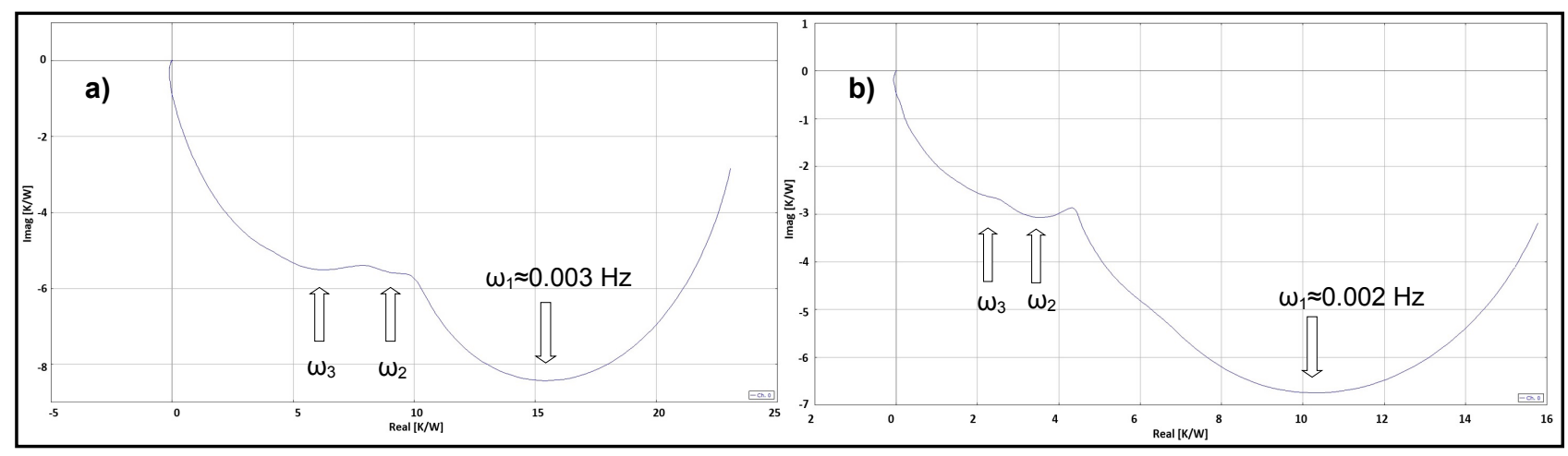

Fig. 6. Nyquist plot of the transfer thermal impedance for a) $2.5 \mathrm{~mm}$ gap core and b) $1.0 \mathrm{~mm}$ gap core

The time constant spectrum (figure 7) can be obtained by deconvolution from the complex thermal impedance [3]. Although the plot appears to shows two time constants it has to be concluded that the time constants $T_{2}$ and $T_{3}$ corresponding to the frequencies $\omega_{2}, \omega_{3}$ are either represented by a single wide-base peak due to the fact that they are closely spaced or the middle one is not shown in the spectrum. The time constant spectrum and the Nyquist plot allow to determine the structure of a component under test and indicate that the inductor used can be modelled, with a very close approximation, as a three-layer structure.

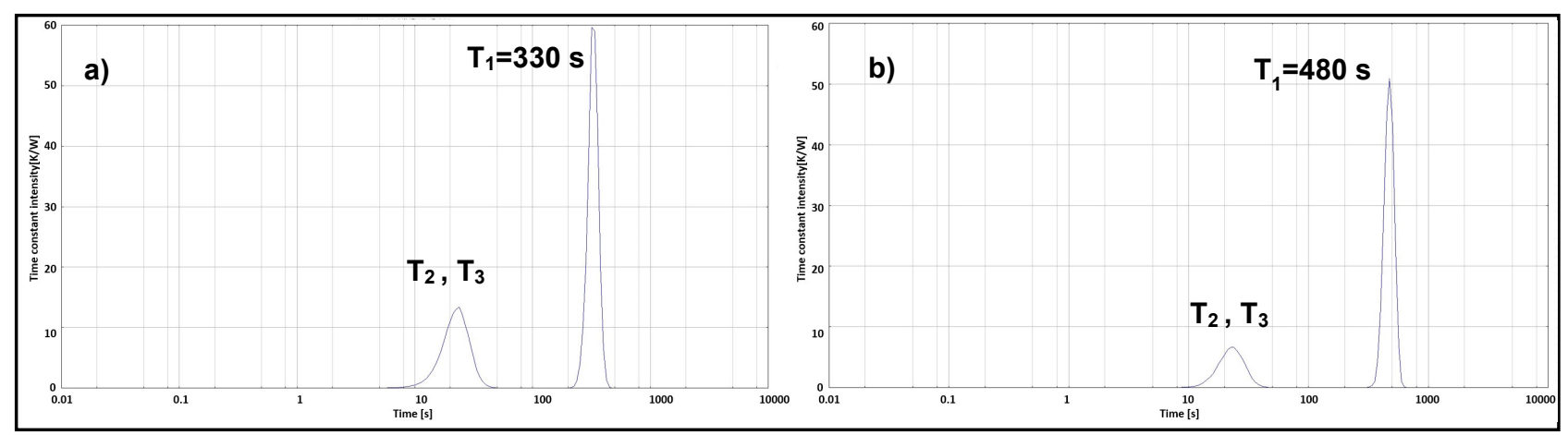

Fig. 7. Time constant distribution for a) $2.5 \mathrm{~mm}$ gap core inductor and b) $1.0 \mathrm{~mm}$ gap core inductor

The data from the experiment were also processed using Matlab functions to obtain their mathematical representation in a form of a transfer function which can be subsequently employed to create Nyquist plots. The transfer function with a fit to the experimental data of more than $99 \%$ and the corresponding frequency response plot of figure 8 show clearly that there are three layers in the heat flow path.

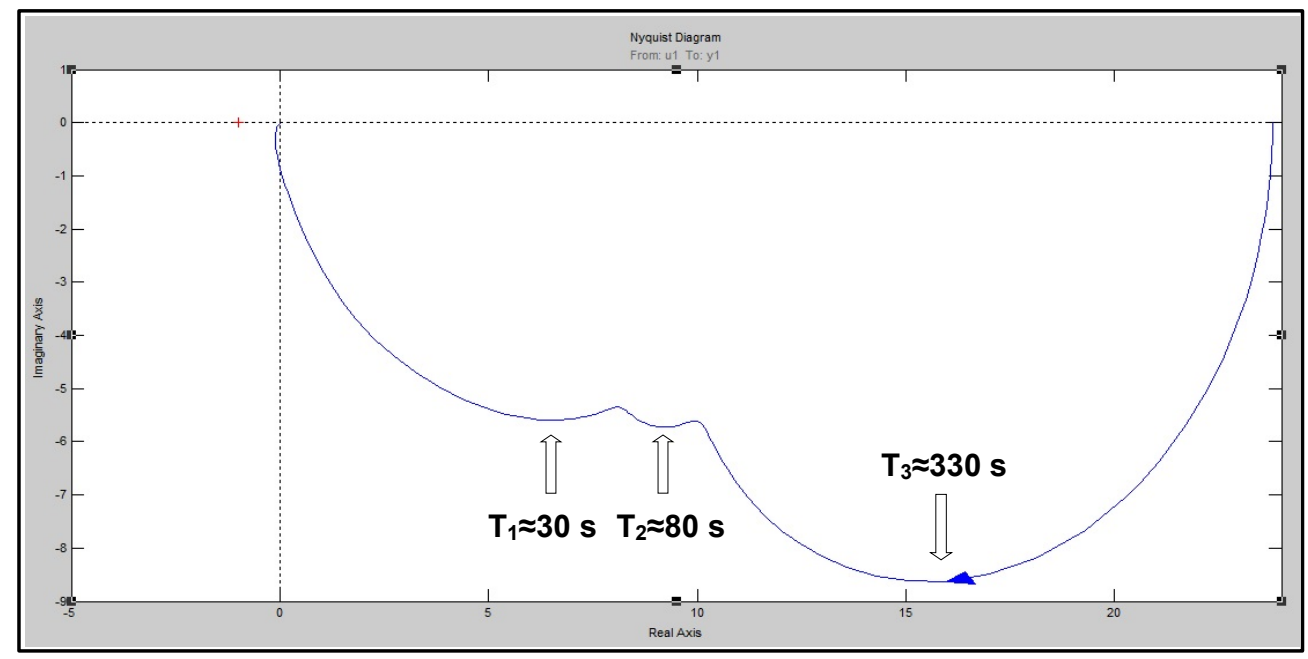

Fig. 8. Nyquist plot of experimental data for $2.5 \mathrm{~mm}$ gap core inductor using Matlab 


\subsection{1/qirt.2016.053}

The structure of the heat flow path can be also represented by the cumulative structure function which is a plot of cumulative capacitance versus cumulative resistance (figure 9). The starting point corresponds to the heat source, the vertical asymptote at the end of $R_{\text {th }}$ relates to the ambient with an infinite thermal capacitance. Steep and flat sections indicate regions well and poorly conductive, respectively.

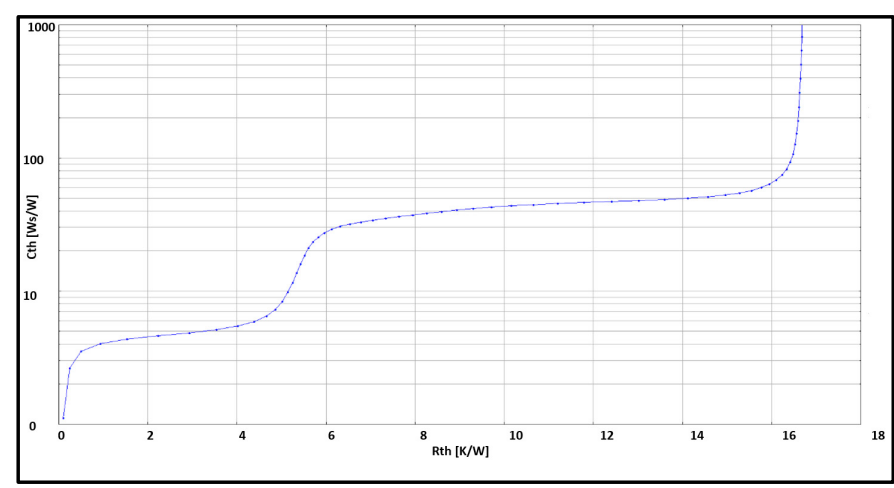

Fig. 9. Cumulative structure function for experimental data

\section{Increase in power loss due to the widening of the air gap}

The origins of losses in magnetic components are traditionally falling into two categories: core losses and copper losses. Ascertainment of the core loss can be accomplished by the means of examining the data provided by the manufacturer for a given core material. The data usually comprise of plots or equations characterising core loss density as a function of the peak value of $A C$ flux density swing $\Delta B$, switching frequency and core temperature. The loss in windings for high frequency magnetic circuits is governed by the following mechanisms: low frequency loss caused by power dissipation in DC resistance of the windings, high frequency loss brought about by eddy currents (the skin and proximity effects) and third one, being the focus of this paper, the fringing-effect loss present in magnetic components with gapped cores. As it is demonstrated below for the given system parameters of the carried out experiment (see figure 10 and table 1) the core loss remained effectively unchanged and the increase in copper loss due to somewhat higher RMS value of the current in the $38.95 \mu \mathrm{H}$ inductor cannot account for the extra power loss, therefore, this has to be attributed to the fringing field of the wider air gap.

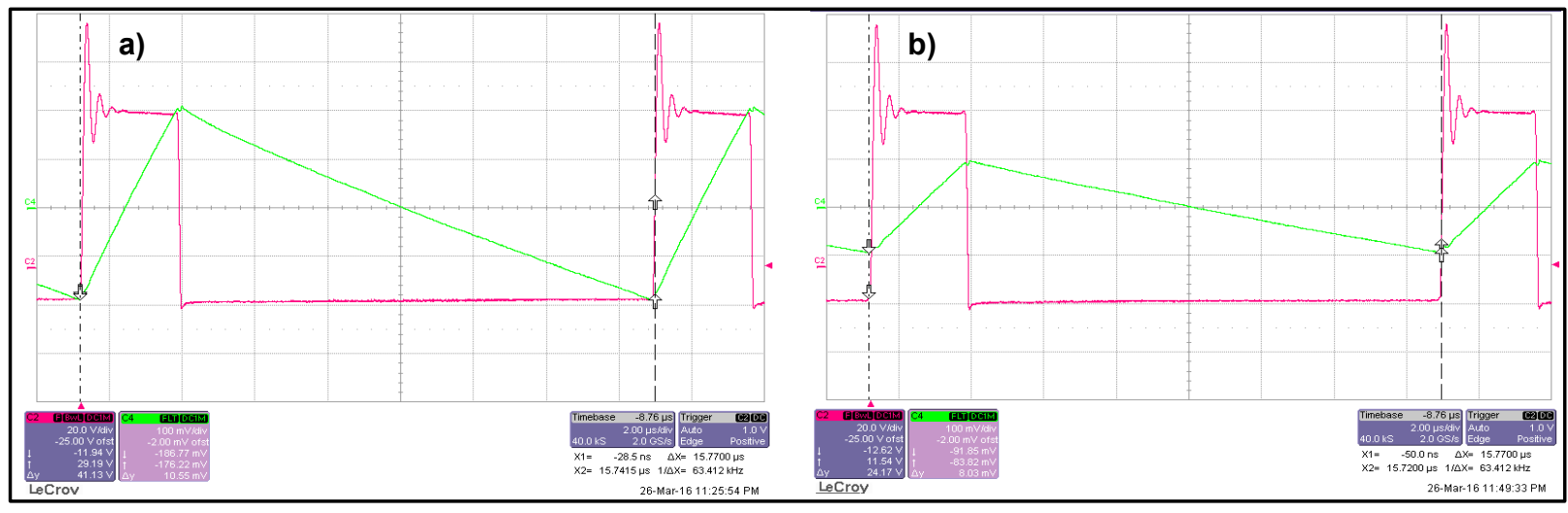

Fig. 10. Voltage waveform (duty cycle) and current ripple for a) $2.5 \mathrm{~mm}$ and b) $1.0 \mathrm{~mm}$ gap inductor

Table 1. System parameters of experiment

\begin{tabular}{|l|l|l|}
\cline { 2 - 3 } \multicolumn{1}{c|}{} & $2.5 \mathrm{~mm}$ gap inductor & $1.0 \mathrm{~mm}$ gap inductor \\
\hline Switching frequency $-\mathrm{f}$ & $63.4 \mathrm{kHz}$ & $63.4 \mathrm{kHz}$ \\
\hline Flux density swing $-\Delta B$ & $0.04 \mathrm{~T}$ & $0.04 \mathrm{~T}$ \\
\hline Current ripple $-\Delta I$ & $4.01 \mathrm{~A}$ & $1.9 \mathrm{~A}$ \\
\hline Duty cycle $-\mathrm{D}$ & $17.5 \%$ & $17.5 \%$ \\
\hline Mean core temp. $-\mathrm{T}$ & $55^{\circ} \mathrm{C}$ & $42{ }^{\circ} \mathrm{C}$ \\
\hline Output voltage $-V_{0}$ & $12 \mathrm{~V}$ & $12 \mathrm{~V}$ \\
\hline Output current $-I_{0}$ & $3 \mathrm{~A}$ & $3 \mathrm{~A}$ \\
\hline
\end{tabular}




\subsection{1/qirt.2016.053}

\subsection{Losses in core material}

For a given magnetic core, manufacturers often represent the total core loss per unit volume in the form of the basic Steinmetz equation; Eq. (2).

$$
P_{v}=k \cdot f^{m} \cdot \Delta B^{n} \cdot\left(c t-c t 1 \cdot T+c t 2 \cdot T^{2}\right)
$$

The manufacturer's plot is obtained for sinusoidal excitation rather than for rectangular voltage waveforms hence the obtained core loss for the output inductor in figure 2 would somewhat differ from the actual value. A practical method for calculating the core losses in magnetic components for an arbitrary shape of the magnetizing current is given in [4]. In this approach the frequency of operation for a non-sinusoidal waveform is replaced with an equivalent sinewave frequency by the means of Eq. (3) for a piecewise linear character of magnetic flux density $B$, where $B_{k}$ and $t_{k}$ denote values and time where a reversal in the magnetic flux density takes place.

$$
f \sin _{e q}=\frac{2}{\pi^{2}} \sum_{k=2}^{K}\left(\frac{B_{k}-B_{k-1}}{B_{\max }-B_{\min }}\right)^{2} \cdot \frac{1}{t_{k}-t_{k-1}}
$$

For the triangular current waveforms of figure 10 with the switching period $T_{o}$ Eq. (3) yields;

$$
f \sin _{e q}=\frac{2}{\pi^{2} \cdot T_{o}} \cdot \frac{1}{D \cdot(1-D)}
$$

The equivalent frequency is inserted into Eq. (2) and together with corresponding manufacturers' parameters for a given magnetic material used to obtain core losses. One can conclude from the Eq. (3) that the power loss density $P_{v}$ shows clear dependence on the duty cycle $D$ and the triangular current waveforms with the duty cycle of about 0.5 exhibit somewhat lower core losses compared with the sinusoidal excitation.

The calculated value of peak $A C$ flux density $\Delta B$ for the inductors used in the experiment is about $0.04 \mathrm{~T}$. The value is to a great extend independent of the change in inductance of the components as the volt-seconds applied to the coils are the same in both cases. Increasing the air gap affects the shape of B-H curve of magnetic circuit bringing about shearing of the $\mathrm{B}-\mathrm{H}$ loop. With no gap present the slope of the curve is as steep as possible, the larger the air gap the lower the slope and the greater value of magnetic field strength $\mathrm{H}$ (proportional to magnetizing current) to reach the same value of B. Thus, the increase in the inductor's current ripple should not alter the peak value of AC flux density swing $\Delta B$.

As mentioned above the converter runs at the frequency of about $63.4 \mathrm{kHz}$ with the duty cycle of $17.5 \%$ which according to Eq. (4) gives:

$$
f \sin _{e q}=88988 \mathrm{~Hz} \text {. }
$$

If this equivalent frequency is used in Eq. (2) instead of the switching frequency the total core loss per unit volume for the constructed inductors is

$$
\begin{gathered}
P_{v 1}=3.2 \cdot f \sin _{e q}{ }^{1.46} \cdot 0.04^{2.75} \cdot\left(2.45-0.031 \cdot 42+0.000165 \cdot 42^{2}\right) \cong 11.09 \frac{\mathrm{kW}}{\mathrm{m}^{3}} \\
P_{v 2}=3.2 \cdot f \sin _{e q}{ }^{1.46} \cdot 0.04^{2.75} \cdot\left(2.45-0.031 \cdot 55+0.000165 \cdot 55^{2}\right) \cong 9.59 \frac{\mathrm{kW}}{\mathrm{m}^{3}}
\end{gathered}
$$

for the inductors with $1 \mathrm{~mm}$ and $2.5 \mathrm{~mm}$ gap, respectively. The discrepancy in the mean core temperatures between the chokes ought to be ascribed to the greater heat radiating from the coil of $2.5 \mathrm{~mm}$ gap core inductor.

In order to obtain the actual core loss these values have to be multiplied by the effective magnetic volume of the core used $V e$. For ER28/14/11 3C90 ferrite cores the Ve value is $5.260 \mathrm{~m}^{3}$. Hence;

$$
\begin{aligned}
& P_{\text {core } 1}=P_{v 1} \cdot V_{e} \cong 0.058 \mathrm{~W} \\
& P_{\text {core } 2}=P_{v 2} \cdot V_{e} \cong 0.05 \mathrm{~W}
\end{aligned}
$$

It should be noted that the above calculations do not take into account the influence of the DC part of the flux density on core losses as manufacturers do not provide data of the effect of a DC-bias on material properties. As shown in [5] this may have a measurable impact on power dissipated in ferrites. 


\subsection{1/qirt.2016.053}

\subsection{Losses in copper}

For high frequency operation current distribution within windings is affected by the skin effect that tends to reduce the net current density in the center of a conductor and the proximity effect responsible for inducing eddy currents in neighbouring conductors. The mechanisms effectively increase the resistance of the wire from its low-frequency value $\mathrm{R}_{\mathrm{DC}}$ to so-called $A C$ resistance $\mathrm{R}_{\mathrm{AC}}$ thus, the copper loss can be expressed in the form of Eq. (5).

$$
P_{\text {copper }}=I_{R M S}^{2} \cdot R_{A C}
$$

The RMS values of the current waveforms illustrated in figure 11 are $3.05 \mathrm{~A}$ and $3.2 \mathrm{~A}$ for $87.08 \mu \mathrm{H}$ and 38.95 $\mu \mathrm{H}$ inductor, respectively.

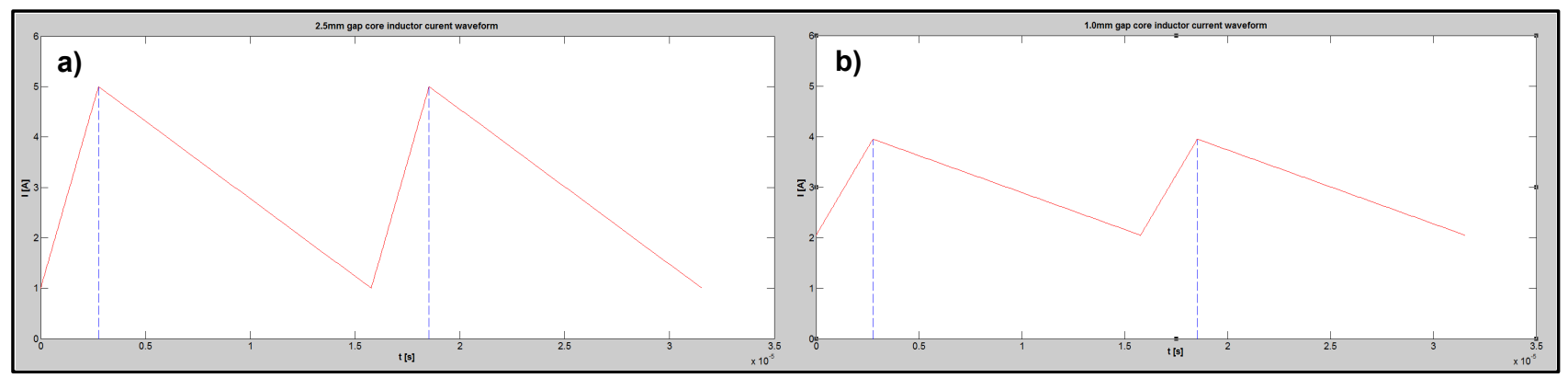

Fig. 11. Current waveform for a) $2.5 \mathrm{~mm}$ gap core inductor and b) $1.0 \mathrm{~mm}$ gap core inductor

Assuming no impact of the wider air gap on the fringing effect, the $R_{A C}$ value should be analogous for both inductors as other parameters such as frequency of operation, configuration of the winding etc. are unchanged and the only alteration in power dissipation in copper is brought about by the somewhat higher RMS values of the inductor current. According to Eq. (5) there is about $10 \%$ increase in the copper loss in the inductor with the wider air gap.

$$
\left.P_{\text {copper }(2.5 \mathrm{~mm})}=I_{R M S(2.5 \mathrm{~mm})^{2}} \cdot R_{A C}=1.1 \cdot I_{R M S(1.0 \mathrm{~mm})}\right)^{2} \cdot R_{A C}=1.1 \cdot(3.05)^{2} \cdot R_{A C}=(3.2)^{2} \cdot R_{A C}
$$

\subsection{Ascertainment of increase in fringing-effect loss}

The total power loss in the inductors was estimated as presented in section 2 of this paper. The outcome of the measurements is as follow:

$$
\begin{aligned}
& P_{\text {TOTAL }(1.0 \mathrm{~mm})}=1.89 \mathrm{~W} \\
& P_{\text {TOTAL }(2.5 \mathrm{~mm})}=2.15 \mathrm{~W}
\end{aligned}
$$

Subtracting the core loss calculated for $1.0 \mathrm{~mm}$ gap core inductor out of its total power loss one can obtain the amount of power dissipated in the windings.

$$
P_{\text {copper }(1.0 \mathrm{~mm})}=1.89-0.058=1.832 \mathrm{~W}
$$

The $10 \%$ increase in the copper loss for the wider air gap inductor yields:

$$
P_{\text {copper }(2.5 \mathrm{~mm})}=1.1 \cdot 1.832=2.015 \mathrm{~W}
$$

The above figure combined with the core loss for $38.95 \mu \mathrm{H}$ inductor cannot account for the total loss in the component. Therefore, it must be the amplified fringing-effect power loss due to the change in the size of the air gap that is behind the extra power dissipated.

$$
\Delta P_{\text {fring-effect }}=P_{\text {TOTAL }(2.5 \mathrm{~mm})}-\left(P_{\text {copper }(2.5 \mathrm{~mm})}+P_{\text {core } 2}\right)=2.15-(2.015+0.05)=0.085 \mathrm{~W}
$$




\subsection{1/qirt.2016.053}

\subsection{Conclusions}

In this paper the impact of the fringing field of an air gap on power loss and temperature distribution in the windings of output inductors in high-frequency forward converters is presented. It has been shown that the centregapped core inductors display a distinctive pattern in temperature distribution in windings if measured along the middle column. The thermographic analysis of the inductor used identified variations in time constants $\mathrm{T}_{1}$ corresponding to heat removal from the surface of the component to the ambient. This almost certainly can be contributed to the observed change in the current ripple i.e. RMS values of the inductor current as well as to variations in the impact of the fringing field on the copper windings thus the lower/higher power dispersion in the coil. The time constant spectrum, the Nyquist plot and the cumulative structure function determined the structure of the magnetic component indicating that it can be modelled as a three-layer construction. The ascertainment of individual power losses due to different power dissipation sources allowed for the extraction of the increase in the fringing-effect loss due to greater fringing field caused by the change in the size of the air gap.

\section{REFERENCES}

[1] Ferroxcube Data Handbook: Soft Ferrites and Accessories, p.493, 2009.

[2] MicReD Hardware Products: Thermal Transient Test and Measurement. http://s3.mentor.com/public_documents/datasheet/products/mechanical/products/t3ster-technical-info.pdf. Accessed - 26.10.2015.

[3] Szekely V.: Identification of rc networks by deconvolution: chances and limits, IEEE Transactions on Circuits and Systems I: Fundamental Theory and Applications, vol. 45, pp. 244 - 258, Mar. 1998.

[4] Albach M., Durbaum Th., Brockmeyer A., Calculating Core Losses in Transformers for Arbitrary Magnetizing Currents. A Comparison of Different Approaches. IEEE, 1996.

[5] Brockmeyer A., "Experimental evaluation of the influence of DC premagnetization on the properties of power electronic ferrites", in IEEE APEC Proc., 1996 